\title{
Intensified synthesis and post-synthetic modification of covalent organic frameworks using a continuous flow of microdroplets technique
}

\author{
Vikram Singh $^{1,2,3}$, Seungwook Jang ${ }^{1,3}$, Niraj K. Vishwakarma ${ }^{1}$ and Dong-Pyo Kim ${ }^{1}$ \\ Covalent organic frameworks (COFs) are two-dimensional/three-dimensional crystalline polymeric materials with diverse molecular \\ backbones and topologies and are strong candidates for numerous applications. However, the ready availability of these materials \\ is a challenge. The key issues are slow production rates, harsher and longer reaction conditions, and a need for post-synthetic \\ modification to obtain desired molecular functionalities. Only a few studies, including those using microfluidic techniques, have \\ focused on refining these factors, but these reports lack synthetic continuity and scalability. Herein, we present a fast, \\ intensified and continuous synthesis and post-synthetic modification of $\beta$-ketoenamine-linked COFs by confining organic building \\ units into moving microdroplets in a transparent capillary. This study introduces a one-step, facile approach to serially modify \\ $\mathrm{NO}_{2}$ - to $\mathrm{NH}_{2}$-substituted COFs in a fraction of the time, effort and cost of traditional methods. These results may stimulate the \\ development of novel COFs with unique chemistries and functions for various applications.
}

NPG Asia Materials (2018) 10, e456; doi:10.1038/am.2017.209; published online 5 January 2018

\section{INTRODUCTION}

Covalent organic frameworks (COFs) are a class of porous, crystalline materials featuring organic building units (OBUs) linked via strong covalent bonds. ${ }^{1-3}$ Recently, COFs have been used in diverse applications, such as $\mathrm{CO}_{2}$ capture, ${ }^{4}$ electrochemical $\mathrm{CO}_{2}$ reduction, ${ }^{5}$ organocatalysis, ${ }^{6}$ photocatalysis, ${ }^{7}$ separation and storage of gases, ${ }^{8,9}$ and sensing, ${ }^{10}$ which makes COFs highly promising futuristic materials. Nevertheless, the construction of new two-dimensional/threedimensional COF structures with high crystallinity, higher surface areas and enhanced stability remains a priority. ${ }^{11-14}$ Likewise, the postsynthetic modification of COFs to tailor the porosity, functionality and surface reactivity is often required to introduce a reactive functional group/moiety, which otherwise is challenging in a single step due to the potential occurrence of side reactions. ${ }^{4,15,16}$

Solvothermal-based strategies extraordinarily enhance the COF synthetic efficiency and quality for intended applications. ${ }^{16-18}$ However, harsh experimental conditions (sealed Pyrex tubes, inert atmosphere, high temperature/pressure and longer reaction times $(72-168 \mathrm{~h})$ ) for the formation of the porous, crystalline covalent network preclude the ready availability and processability of COFs while impeding scalability. ${ }^{2,17}$ Additional post-synthetic modification step(s) with time-consuming purifications and separations are often required. ${ }^{18,19}$ Alternate synthetic approaches are scarce, except for microwave-assisted synthesis, ${ }^{20,21}$ which has rather inconsistent synthetic control; mechanosynthesis, ${ }^{22}$ which affords COFs with relatively low crystallinity; and a few flow-based approaches, which lack longterm scalability owing to potential channel blockage issues with time. In particular, there are no reports on performing serially intensified synthesis and post-synthetic modification of COFs.

The continuous flow of microdroplets (CFM) technique in microfluidic channels to encapsulate molecular-building units has enabled the production of several inorganic materials, such as metal organic frameworks, ${ }^{23,24}$ zeolites, $^{25}$ metal nanocrystals ${ }^{26}$ and silica nanoparticles, ${ }^{27}$ with higher space-time yields and qualities. The advantage of this process lies in the advection-based mixing and minimal contact of the 'in-droplet'-generated material with the channel surface even at a high concentration of growing particles. $^{23,28}$ This process is unlike simple continuous processes, which are prone to channel clogging at extended periods, especially when the growing particles (such as COFs) are insoluble in most solvents. ${ }^{29}$ On the basis of these observations, we report an alternative strategy for the serially intensified and continuous synthesis and postsynthetic modification of $\beta$-ketoenamine-linked COFs based on a CFM approach in a transparent polytetrafluoroethylene capillary. $\beta$-Ketoenamine-linked COFs were chosen due to their exceptional stability under varying chemical and thermal stresses, unlike imine-

${ }^{1}$ Center of Intelligent Microprocess of Pharmaceutical Synthesis, Department of Chemical Engineering, Pohang University of Science and Technology (POSTECH), Pohang, South Korea

${ }^{2}$ Current address: Centre for Nanoscience and Nanotechnology, Panjab University, Chandigarh, 160015, India.

3These authors contributed equally to this work.

Correspondence: Professor D-P Kim, Center of Intelligent Microprocess of Pharmaceutical Synthesis, Department of Chemical Engineering, Pohang University of Science and Technology (POSTECH), Room 304, Environmental Engineering Building 77, Cheongam-ro, Nam-gu, Pohang 37673, South Korea.

E-mail: dpkim@postech.ac.kr

Received 13 July 2017; revised 29 September 2017; accepted 12 October 2017 
and/or boronic acid-based COFs, which directly affects their applicability. This high stability arises from the irreversible nature of the imine-to-enamine tautomeric step, which is attributable to the reasonably large basicity of the $-\mathrm{NH}$ group and then the phenolic $-\mathrm{OH}$ group, leading to a stable $\beta$-ketoenamine linkage. ${ }^{30}$

\section{MATERIALS AND METHODS}

1,3,5-Triformylphloroglucinol (Tp) was synthesized using a known procedure. $^{22}$ All other chemicals, including reagents and solvents were commercially available and procured either from Sigma-Aldrich (Seoul, Korea), Alfa-Aesar (Seoul, Korea) or TCI Chemicals (Tokyo, Japan). All reagents were used as received without any further purification. All solvents received were dried and used under an inert gas atmosphere. All liquid-state ${ }^{1} \mathrm{H}$ nuclear magnetic resonance (NMR) spectra were recorded using a Bruker (Billerica, MA, USA) AVANCE III $300 \mathrm{MHz}$ FT-NMR spectrometer. All solid-state ${ }^{13} \mathrm{C}$ and ${ }^{15} \mathrm{~N}$ NMR spectra (cross-polarization magic angle spinning were recorded using a Bruker AVANCE $\mathrm{II}^{+} 400 \mathrm{MHz}$ solid-state NMR (ssNMR) spectrometer. The powder X-ray diffraction (PXRD) patterns were recorded on a highresolution powder X-ray diffractometer (SmartLab, Rigaku, The Woodlands, TX, USA) with a $\mathrm{Cu} \mathrm{K \alpha}$ radiation source (wavelength $=1.5405 \AA$ ) operated at $30 \mathrm{kV}$ and a power of $30 \mathrm{~mA}$ utilizing a HyPix 3000 detector. The data were collected within the $2 \theta$ range of $4-40^{\circ}$. Solid-state ultraviolet-visible (UV-vis) spectra were recorded on a Shimadzu (Kyoto, Japan) UV-2501PC UV-vis spectrophotometer equipped with a $50 \mathrm{~mm}$ integrating sphere. $\mathrm{BaSO}_{4}$ was used as a white standard reference. Diffuse reflectance spectral data were transformed into absorbance using the Kubelka-Munk function. Fourier transform infrared spectra were recorded in the wavenumber range of $650-4000 \mathrm{~cm}^{-1}$ using a JASCO (Tokyo, Japan) FT/IR-4600 instrument equipped with a universal ZnSe ATR accessory. Thermogravimetric analysis (TGA) was carried out on a TA Instruments model SDT Q600 instrument under a $\mathrm{N}_{2}$ atmosphere at a heating rate of $10^{\circ} \mathrm{C} \mathrm{min}-1$ within a temperature range of $30-800{ }^{\circ} \mathrm{C}$. Scanning electron microscopy (SEM, North Billerica, MA, USA) images were obtained with a Philips (Amsterdam, Netherlands) XL30S FEG FE-SEM instrument with a tungsten filament as an electron source operated at $5 \mathrm{kV}$. The samples were sputtered with $\mathrm{Pt}$ before imaging. High-resolution transmission electron microscopy (TEM) images were obtained on a JEOL JEM-2100F instrument at an accelerating voltage of $200 \mathrm{kV}$ and were manipulated using Gatan Micrograph software (Pleasanton, CA, USA). TEM samples were prepared by drop-casting the sample from an isopropanol solution onto TEM copper grids (200 mesh) provided by Electron Microscopy Sciences (Hatfield, PA, USA). $\mathrm{N}_{2}$ adsorption-desorption isotherms were performed using Autosorb-iQ and Quardsorb SI instruments from Quantachrome (Boynton Beach, FL, USA). Inductively coupled plasma atomic emission spectroscopy measurements were performed using a Perkin Elmer (Waltham, MA, USA) Optima8 300 instrument with a radio-frequency power of $1300 \mathrm{~W}$, plasma flow of $121 \mathrm{~min}^{-1}$, auxiliary flow of $0.2 \mathrm{l} . \mathrm{min}$, nebulizer flow of $0.61 \mathrm{~min}^{-1}$ and a segmented-array charge-coupled device detector.

a<smiles>[R6]c1cc(N)c(Br)cc1N</smiles>

$2 \times$<smiles>O=Cc1c(O)c(C=O)c(O)c(C=O)c1O</smiles>
Organic Building Units (OBUS)

\section{R}<smiles>[R]C=C(C)N=Cc1c(O)c(C(C)(C)C)c(O)c(CCC)c1C(C)C</smiles>

Imine-form of COFs

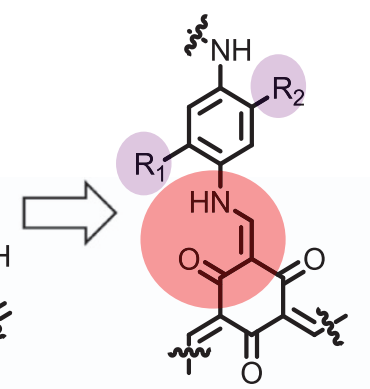

$\beta$-ketoenamine-form of COFs
TpPa-H

$\mathrm{R}_{1}=\mathrm{R}_{2}=\mathrm{H}$

TpPa-Me

$\mathrm{R}_{1}=\mathrm{R}_{2}=\mathrm{CH}_{3}$

$\mathrm{TpPa}-\mathrm{NO}_{2}$

$\mathrm{R}_{1}=\mathrm{NO}_{2}, \mathrm{R}_{2}=\mathrm{H}$

b

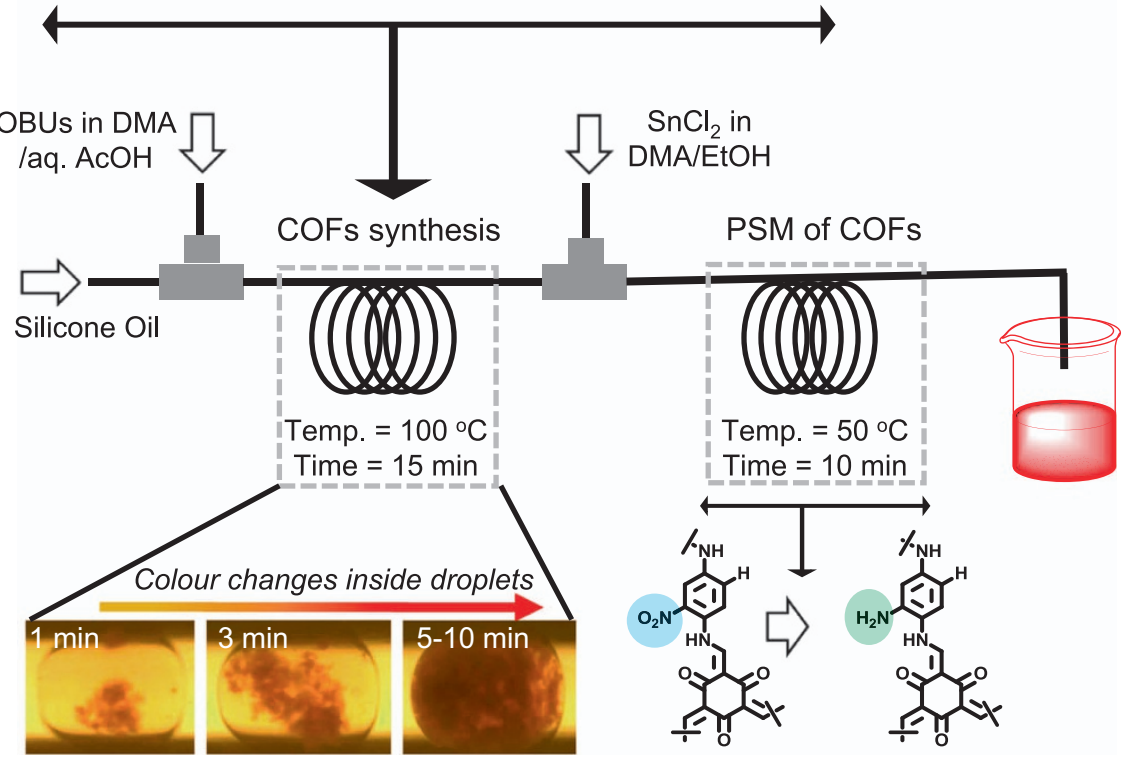

Figure 1 (a) Schematic representation of the covalent organic framework (COF) synthesis through initial imine formation and subsequent tautomerization to give the $\beta$-ketoenamine-linked COFs; (b) representation of the synthesis of the COFs ( TpPa-H, TpPa-Me and TpPa- $\mathrm{NO}_{2}$ ) and serial post-synthetic modification of $\mathrm{TpPa}-\mathrm{NO}_{2}$ to $\mathrm{TpPa}_{-} \mathrm{NH}_{2}$; (c) monitoring the 'in-droplet' reaction progress $\left(15 \mathrm{~min}, 100{ }^{\circ} \mathrm{C}\right)$ using dynamic color changes and filling of the droplet with a deep-red precipitate. Tp, 1,3,5-triformylphloroglucinol. 


\section{COF synthesis using a CFM technique}

The OBUs, Tp $\left(\mathrm{D}_{3 \mathrm{~h}}\right)$ and the individual diamine $(\mathrm{Pa})$ (that is, 1,4-phenylenediamine $\left(\mathrm{Pa}-\mathrm{H}, \mathrm{D}_{2 \mathrm{~h}}\right), 2,5$-dimethyl-1,4-phenylenediamine $\left(\mathrm{Pa}-\mathrm{Me}, \mathrm{D}_{2 \mathrm{~h}}\right)$ or 2-nitro-1,4-phenylenediamine $\left(\mathrm{Pa}-\mathrm{NO}_{2}, \mathrm{C}_{1 \mathrm{~h}}\right)$ ) in a 2:3 molar ratio were partially dissolved in $2 \mathrm{ml}$ of a dimethylacetamide (DMA) and water mixture (9:1, v/v) for the synthesis of COFs with three different substitutions (TpPa-H, TpPa-Me and $\mathrm{TpPa}-\mathrm{NO}_{2}$, respectively). Thereafter, $200 \mu \mathrm{l}$ of $6 \mathrm{M}$ aqueous acetic acid was slowly added to the mixture to pre-mix the OBUs. This partially solubilized mixture was carefully taken in a $10 \mathrm{ml}$ syringe and quickly fed into a T-junction through polytetrafluoroethylene tubing with a flow rate varying from 20 to $80 \mu \mathrm{min}^{-1}$ using a Harvard Model PHD 2000 syringe pump (Holliston, MA, USA). In another syringe, silicone oil/FC-40 oil was utilized as a carrier fluid. Note that the DMA/water mixture has negligible solubility in silicone oil even at elevated temperatures. Silicone oil was flown at a higher rate $\left(60-240 \mu \mathrm{lmin}^{-1}\right)$ proportional to the flow rate of the pre-mixed OBUs. At the T-junction, facile droplet generation occurred, and the droplets were then carried by the coiled polytetrafluoroethylene tubing, which was immersed in the silicon oil bath kept at a desired temperature $\left(80-100{ }^{\circ} \mathrm{C}\right)$. Optimization of the flow rates and characterization details indicated that a residence time of $15 \mathrm{~min}$ was suitable to obtain high-quality COF products. However, lower residence times resulted in the presence of unreacted and oligomeric materials in the COF pores. Therefore, the reaction time was equivalent to the residence time of the droplets in the heating bath. The droplets were imaged using an optical microscope at time points of 1,3 and 5-10 min, where the droplets became filled with solid material between 5 and $10 \mathrm{~min}$. The droplets were collected at different time intervals (after 5, 10 and $15 \mathrm{~min}$ ), washed with $n$-hexane to remove silicone oil, sequentially washed with $10 \mathrm{ml}$ each of dry DMA, acetone and DCM, and vacuum-dried at $150^{\circ} \mathrm{C}$ for $12 \mathrm{~h}$ to obtain the deep-red-colored COFs.

\section{Serial post-synthetic modification of the COFs}

The TpPa- $\mathrm{NO}_{2}$ droplets exiting the heating bath in the first step were merged with the incoming solution of anhydrous $\mathrm{SnCl}_{2}$ in DMA/ethanol at the second $\mathrm{T}$-junction. The flow rates were adjusted for efficient mixing as much as possible. The combined droplets were moved through a heating bath maintained at $50{ }^{\circ} \mathrm{C}$ for a residence time of $10 \mathrm{~min}$, collected over dry $n$-hexane and subsequently washed with $5 \mathrm{ml}$ each of dry $n$-hexane and DMA and $20 \mathrm{ml}$ of acetone. After drying, the reddish-brown powder was treated with $5 \mathrm{ml}$ of $1 \mathrm{~m} \mathrm{HCl}$ for $10 \mathrm{~min}$. Further washing with $30 \mathrm{ml}$ of $1 \mathrm{M} \mathrm{HCl}$, a copious amount of water and $20 \mathrm{ml}$ of acetone, and vacuum drying at $150{ }^{\circ} \mathrm{C}$ provided activated $\mathrm{TpPa}-\mathrm{NH}_{2}$.

\section{Catalytic performance of $\mathrm{Pd}(\mathrm{OAc})_{2}-\mathrm{TpPa}-\mathrm{H}(1)$}

In a typical catalytic reaction, $0.5 \mathrm{~mol} \%$ of $\mathrm{Pd}(\mathrm{OAc})_{2}-\mathrm{TpPa}-\mathrm{H}(\mathbf{1})$ was added to a mixture of a $1.0 \mathrm{mmol}$ of a suitable aryl halide, $1.5 \mathrm{mmol}$ of phenylboronic acid and $2.0 \mathrm{mmol}$ of potassium carbonate in $5 \mathrm{ml}$ of $p$-xylene and refluxed at $150{ }^{\circ} \mathrm{C}$ for $4 \mathrm{~h}$. Thereafter, the reaction mixture was centrifuged, and the solid material was washed with dichloromethane $(30 \mathrm{ml} \times 3)$. The remaining solid contained the catalyst. The collected organic fractions were combined, washed with water $(20 \mathrm{ml} \times 3)$ and evaporated under reduced pressure to obtain the crude product. Chromatographic purification over silica gel yielded the pure product in $>90 \%$ yield. Catalyst recycle tests were performed under similar conditions as above using $p$-methoxybromobenzene and phenylboronic acid as coupling partners. The catalyst was recovered by centrifugation, consecutively washed with dichloromethane and methanol, dried and used as the catalyst for the next cycle. The initial Pd content was found to be $7.06 \mathrm{wt} \%$, and after three cycles, the Pd content decreased to $6.77 \mathrm{wt} \%$, as determined by inductively coupled plasma atomic emission spectroscopy.

\section{COF stability tests}

Thermal stability. To ascertain the extents of structural stability and decomposition patterns of COFs and their usability at elevated temperatures, the COFs were subjected to increasing temperatures ranging between 30 and $800{ }^{\circ} \mathrm{C}$ under a $\mathrm{N}_{2}$ atmosphere with heating steps of $10^{\circ} \mathrm{C} \mathrm{min}^{-1}$ using the TGA instrument.

Temporal stability. The COFs were kept in open air for 2 weeks at room temperature to determine their extents of degradation under atmospheric conditions. For this purpose, solid-state UV-vis spectra were recorded after the synthesis, at the end of the first week and at the end of the second week.

Chemical stability. To determine the stabilities of the COFs under chemical stress, the COFs were dispersed in various solvents, including water, DCM, hexane and $9 \mathrm{~m} \mathrm{HCl}$ for $48 \mathrm{~h}$ at room temperature. Afterwards, they were subjected to a purification process and activated at $150^{\circ} \mathrm{C}$ under vacuum before subsequently measuring their respective PXRD patterns.

\section{RESULTS AND DISCUSSION}

Three $\beta$-ketoenamine-linked COFs with different substitutions, namely, TpPa-H, TpPa-Me and $\mathrm{TpPa}-\mathrm{NO}_{2}$ (Figure 1), were synthesized using a CFM technique under 'microfluidic-solvothermal' conditions. $^{22,31}$ In a typical procedure, a solution containing $0.08 \mathrm{M}$ $\mathrm{Tp}$ and $0.12 \mathrm{~m}$ the corresponding $p$-diamine in $2 \mathrm{ml}$ of a $N, N$-DMA and water mixture $(9: 1, v / \mathrm{v})$ and $6 \mathrm{M}$ aqueous acetic acid (Supplementary Table S1 and Supplementary Figure S1) was first fed into a T-micromixer through a $0.01 \mathrm{~m}$ straight capillary. Silicone/ FC-40 oil, utilized as a carrier fluid, enabled smooth microdroplet generation with a lateral diameter of $\sim 200 \mu \mathrm{m}$ at the T-micromixer with judiciously optimized flow rates $(\mathrm{d} V / \mathrm{d} t)$. A 1:3 flow rate ratio of the OBUs to oil was found to be optimum. The OBU-filled microdroplets moved through the coiled capillary maintained at an elevated temperature $\left(80-100^{\circ} \mathrm{C}\right)$ for a residence time of $5-15 \mathrm{~min}$ $\left(t_{\mathrm{r}}\right)$. 'By-eye' visualization of the dynamic color changes (yellow $\rightarrow$ orange $\rightarrow$ red) assisted in monitoring the reaction progress over time, where the initial OBUs transformed into oligomers (yellow-orange) and finally into the CFM-based COFs (deep red). ${ }^{32}$

Apparently, the reaction conditions prohibited separate injection of the OBUs through two inlets, as this invariably led to the deposition of solid material at the T-junction at extended periods, likely due to unrestrained, violent mixing and direct contact with the channel surface. The optimized DMA/ $\mathrm{H}_{2} \mathrm{O}(9: 1, \mathrm{v} / \mathrm{v})$ content intentionally allowed only partial solubility of the pre-mixed OBUs at room temperature to enable initial 'error-correction' akin to solvothermal conditions. Note that the choice of solvent was based on the partial solubility of at least one reactant at room temperature. Finally, we observed a stable flow of droplets with no difficulty in back pressure regulation, likely due to the suppressed volatility of DMA even at $\sim 100^{\circ} \mathrm{C}$. Other tested solvents, that is, mesitylene, toluene, dioxane and benzene, could not sustain smooth droplet generation and flow due to violent motion and their undesirable solubility in silicone oil, which resulted in material deposition on the channel surface. Under these conditions, we achieved a remarkable continuous production rate in excess of $40 \mathrm{mg} \mathrm{h}^{-1}$. Notably, the continuous flow process can be scaled-up further by constituting parallel channels or by stacking a number of reactors. ${ }^{33-36}$ In addition, we tested our approach vis-à-vis with recent regular continuous flow reports for imine-based $\mathrm{COFs}^{37}$ and boronate-ester-based COF films, ${ }^{38}$ but longer reaction times ( $>1 \mathrm{~min}$ ), even at room temperature, clogged the transparent capillary with bulk COF powder. Moreover, the tautomeric step did not appear to materialize in the given timeframe, thereby making regular flow methods untenable for the $\beta$-ketoenamine-linked COFs.

The reaction progress at $100{ }^{\circ} \mathrm{C}$ was established via optical imaging of the microdroplets, the concomitant color changes and ATR-IR (Attenuated total reflectance-Infrared) analysis. For TpPa-Me, the droplets completely grew within 5-10 min (Figure 1c), and the collected orangecolored material exhibited ATR-IR resonances of $\mathrm{C}=\mathrm{O}$ at $1620 \mathrm{~cm}^{-1}$, $\mathrm{C}=\mathrm{N}$ at $1690 \mathrm{~cm}^{-1}$ and phenolic $\mathrm{O}-\mathrm{H}$ at $\sim 3000 \mathrm{~cm}^{-1}$ (at $5 \mathrm{~min}$ ) related to either imine-based oligomeric species or the COF with trapped/unreacted OBUs (Supplementary Figure S2A and B). In 
contrast, after $15 \mathrm{~min}$ of reaction, the color changed to deep red, and new ATR-IR resonances related to the enamine linkage, that is, $\mathrm{C}=\mathrm{C}$ at $1580 \mathrm{~cm}^{-1}$ and $\mathrm{C}-\mathrm{N}$ at $1250 \mathrm{~cm}^{-1}$, were observed (Figure 2a). The broadened $\mathrm{C}=\mathrm{C}$ absorption likely implied overlap with the lower-energy $\beta$-keto $(\mathrm{C}=\mathrm{O})$ absorption. In addition, TpPa-Me showed a typical methyl C-H absorption at $2920 \mathrm{~cm}^{-1}$, and $\mathrm{TpPa}-\mathrm{NO}_{2}$ showed specific $\mathrm{NO}_{2}$ symmetric and asymmetric absorptions at 1346, 1445 and $1523 \mathrm{~cm}^{-1}$. Lower reaction temperatures $\left(<100^{\circ} \mathrm{C}\right)$ and shorter droplet residence times $(<10 \mathrm{~min}$ ) led to incomplete reactions, as confirmed by ATR-IR analysis (Supplementary Figure S2). ${ }^{13} \mathrm{C}$ cross-polarization magic angle spinning ssNMR spectroscopy of the powders obtained with a 15 min reaction time at $100{ }^{\circ} \mathrm{C}$ revealed peaks consistent with the enamine carbon $(=\mathrm{C}-\mathrm{NH})$ at $\sim 145$ p.p.m. (Figure $2 \mathrm{~b}$ ), methyl carbon $\left(-\mathrm{CH}_{3}\right)$ for TpPa-Me at $\sim 17$ p.p.m. and $\beta$-keto-carbon $(-\mathrm{C}=\mathrm{O})$ at $\sim 182$ p.p.m., along with the notable absence of the imine carbon ($\mathrm{C}=\mathrm{N}$ ) resonance at $\sim 165$ p.p.m. These analyses reveal that the advection-based heat and mass transfer in the moving droplets effectively facilitated the initial reversible imine formation and then locking of the $\beta$ ketoenamine tautomeric form throughout the framework.

The PXRD patterns for the COFs synthesized at $100^{\circ} \mathrm{C}$ were recorded at droplet residence times of 10 and 15 min (Supplementary Figure S3). The PXRD patterns at 15 min displayed characteristically intense peaks at $\sim 5.0^{\circ}$ and relatively less intense but somewhat broad
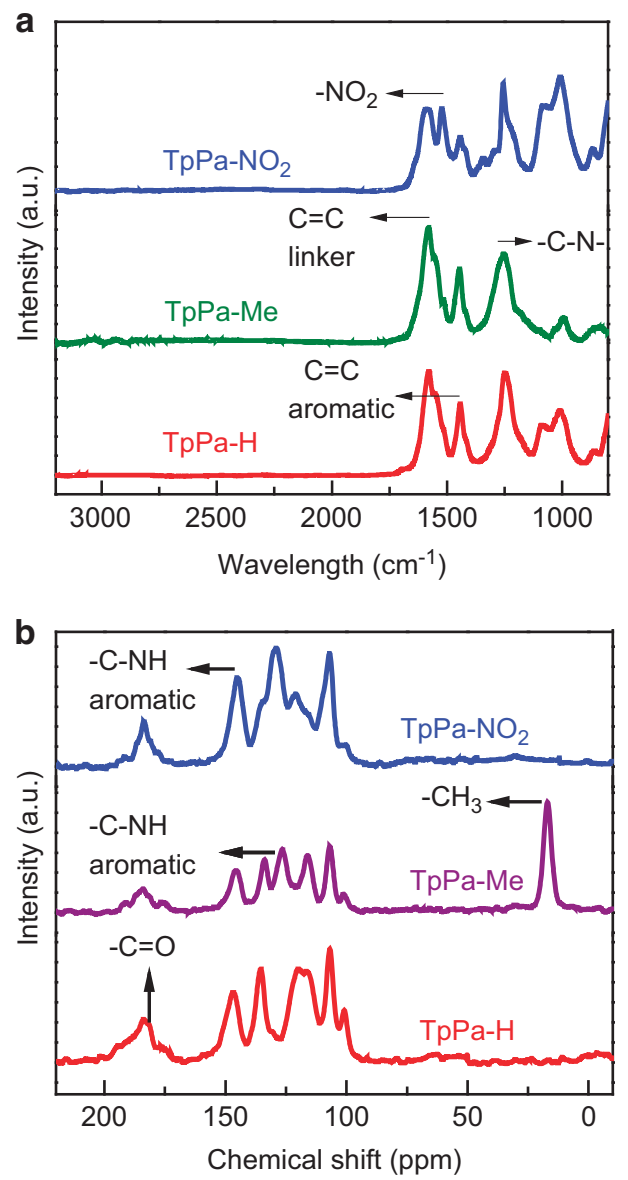

Figure 2 Spectra of the covalent organic frameworks obtained by the indroplet approach at $100{ }^{\circ} \mathrm{C}$ for 15 min reactions. (a) ATR-IR spectra clearly indicating the absence of the organic building units and oligomers; the $y$ axis is \%transmittance; (b) ${ }^{13} \mathrm{C}$ cross-polarization magic angle spinning solidstate nuclear magnetic resonance spectra displaying the characteristic $\beta$-keto peaks at $~ 180$ p.p.m. peaks at $\sim 8^{\circ}, \sim 12^{\circ}$ and $25-28^{\circ}$, corresponding to the (100), (110), (210) and (001) reflection planes, respectively. These reflections are comparable to previously reported solvothermal-based COFs and the simulated patterns (Supplementary Figure S4). 22,31,39 However, these features were not observed at $10 \mathrm{~min}$. Note that the broadness of the PXRD peaks for the $\beta$-ketoenamine-linked COFs is a well-established phenomenon, which likely originates from stacking faults or dislocations, displaying an inter-layer distance estimated at $\sim 3.4 \AA .{ }^{40} \mathrm{It}$ is also noteworthy that this series of COFs has not yet shown very high crystallinity in comparison to COFs with reversible imine or boronateester linkages. ${ }^{41}$ The high-resolution TEM images revealed parallelaligned, stacked sheets ${ }^{40}$ composed of crystalline domains with sizes of 10-40 nm (Supplementary Figures S5 and S6). Accordingly, we observed improved nanoscale crystallinity with slightly enhanced short-range ordering, when compared to the $\beta$-ketoenamine-linked COFs obtained by conventional solvothermal methods. SEM revealed a spherical morphology with a spinous surface, a known feature of this class of COFs (Supplementary Figure S7). Subsequently, we monitored the thermal, temporal and chemical stabilities of the COFs. TGA showed sufficient thermal stability up to at least $300^{\circ} \mathrm{C}$ under nitrogen (Supplementary Figure S8). The nature of the TGA curve and decomposition pattern was found to be substituent-dependent. For $\mathrm{TpPa}-\mathrm{Me}$ and $\mathrm{TpPa}-\mathrm{NO}_{2}$, the methyl/nitro groups decompose before the actual decomposition of the entire framework, which is also in agreement with the solvothermal-based reports. ${ }^{22,31}$ Furthermore, when kept in open air, these COFs were found to be temporally stable for at least 14 days, as evidenced by solid-state UV-vis spectra (Supplementary Figure S9). The photoabsorption profiles of these COFs revealed an absorption edge at $500-550 \mathrm{~nm}$ and were found to be dependent on the nature of the substitution. For instance, for the neutral $(\mathrm{Pa}-\mathrm{H})$ and electron-donating $(\mathrm{Pa}-\mathrm{Me})$ substituents, the solidstate UV-vis spectra of the COFs were redshifted in comparison to the electron-withdrawing $\mathrm{Pa}-\mathrm{NO}_{2}$. The chemical stabilities at ambient room temperature were confirmed by their PXRD patterns, which remained intact even when the COFs were exposed to water, organic solvents and highly acidic $(9 \mathrm{M} \mathrm{HCl})$ conditions for at least $48 \mathrm{~h}$ (Supplementary Figure S10). These results are indicative of the persistent order across the framework, where stable $\pi-\pi$ stacking interactions drive the COF crystallization under 'droplet-solvothermal' conditions and maintain the structural integrity under harsh conditions.

After collecting substantial amounts of the COFs, we performed

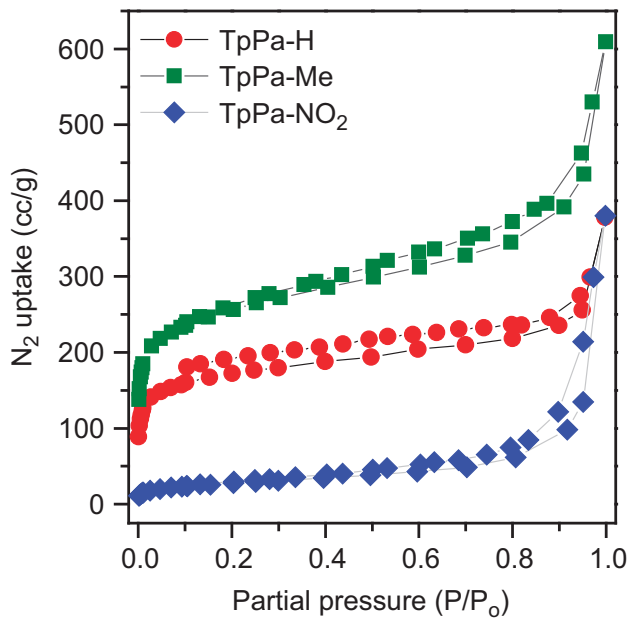

Figure $3 \mathrm{~N}_{2}$ sorption isotherms at $77 \mathrm{~K}$ for the post-treated covalent organic frameworks synthesized at $100{ }^{\circ} \mathrm{C}$ for 15 min reactions. 
a
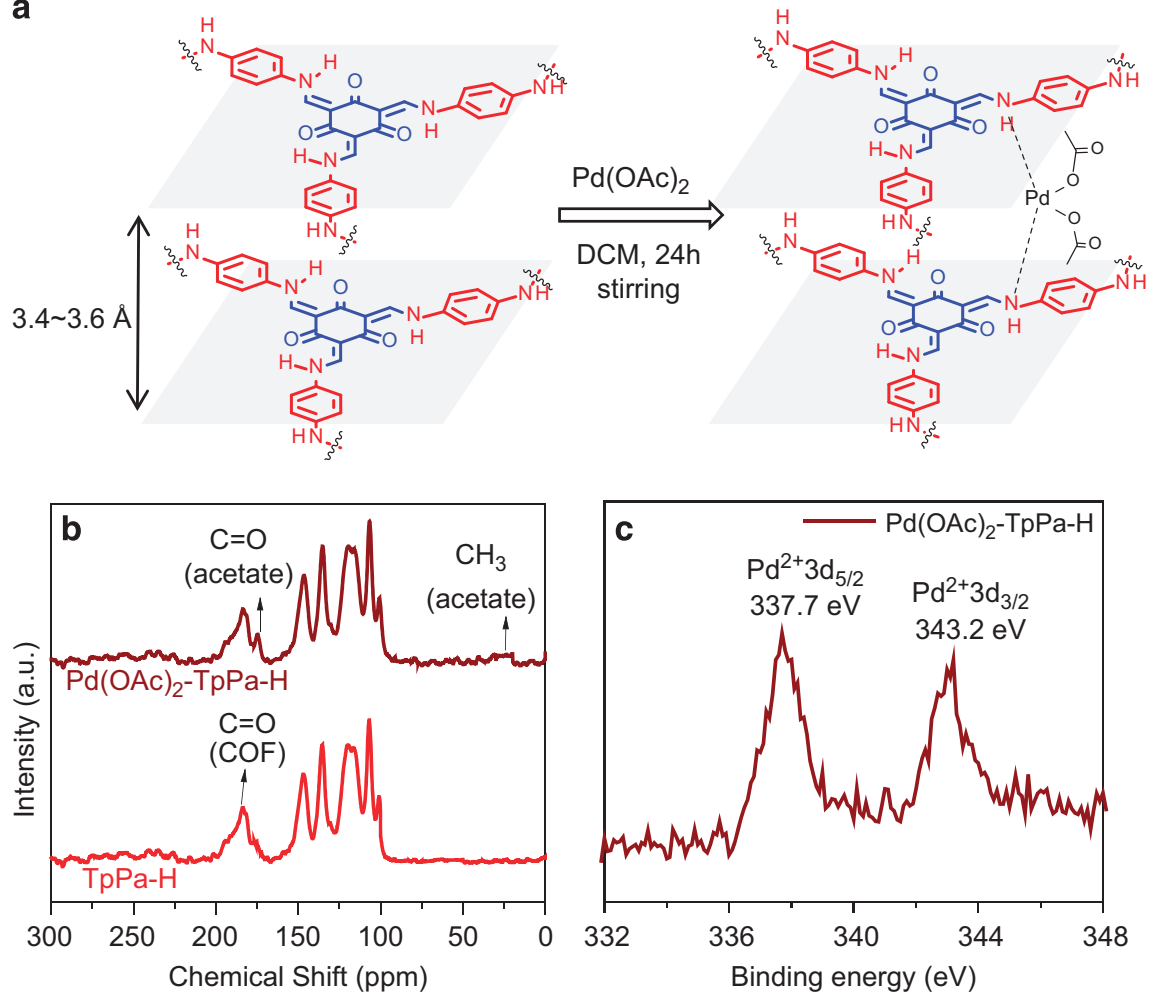

Figure 4 (a) Depiction of $\mathrm{Pd}(\mathrm{OAc})_{2}$ coordination to the TpPa- $\mathrm{H}$ layers leading to the formation of $\mathrm{Pd}(\mathrm{OAc})_{2}-\mathrm{TpPa}-\mathrm{H}(\mathbf{1})$; (b) ${ }^{13} \mathrm{C}$ cross-polarization magic angle spinning solid-state nuclear magnetic resonance spectra of TpPa-H and 1; (c) X-ray photoelectron spectroscopy (XPS) spectrum of 1 revealing characteristic peaks at 337.7 and $343.2 \mathrm{eV}$, corresponding to the $3 d_{5 / 2}$ and $3 d_{3 / 2}$ states of $\mathrm{Pd}(\mathrm{II})$. Tp, 1,3,5-triformylphloroglucinol.

simple post-treatment process to study its impact on the COF characteristics, that is, the structural integrity and microscopic effects. ${ }^{42}$ Herein, the process included heating the powder material at $120^{\circ} \mathrm{C}$ in water, then filtering and drying under vacuum. The posttreatment duration was optimized based on the steric properties of the substituents, that is, $\sim 1 \mathrm{~h}$ for $\mathrm{TpPa}-\mathrm{NO}_{2}$ and a few minutes for $\mathrm{TpPa}$ H. Notably, extended post-treatment did not yield any better results. Nevertheless, we did not observe any remarkable changes to the ${ }^{13} \mathrm{C}$ ssNMR spectra (Supplementary Figure S11) and TGA curves (Supplementary Figure S8) of the COFs after post-treatment, indicating that their chemical functionalities were intact. The noted irreversibility of the tautomeric step makes 'error-correction' unlikely in terms of chemical modification. However, the high-resolution TEM images showed considerable alterations, where TpPa-H showed a flower-like morphology with petals with a sheet-like structure, consistent with literature reports, ${ }^{31,32}$ while $\mathrm{TpPa}-\mathrm{Me}$ and $\mathrm{TpPa}$ $\mathrm{NO}_{2}$ exhibited aligned stacked sheets and crystalline domains with sizes of $20-60 \mathrm{~nm}$ (Supplementary Figure S12). These changes are indicative of layer restacking or a reduction in stacking faults leading to enhanced ordering. Then, we performed $\mathrm{N}_{2}$ adsorption-desorption studies at $77 \mathrm{~K}$ on the post-treated COFs, which exhibited typical type I adsorption isotherms with Brunauer-Emmett-Teller (BET) surface areas $\left(S_{\mathrm{BET}}\right)$ of 632,934 and $167 \mathrm{~m}^{2} \mathrm{~g}^{-1}$ for TpPa-H, TpPa-Me and $\mathrm{TpPa}-\mathrm{NO}_{2}$ (Figure 3), respectively, which are indicative of microporous structures with average pore sizes of $\sim 1.8 \mathrm{~nm}$ (Supplementary Figure S13). The $S_{\mathrm{BET}}$ values are comparable or even larger than those obtained from solvothermal methods or mechanosynthesis. ${ }^{31,32}$

We also monitored the substituent $\left(R_{1} / R_{2}\right)$ effects on the COF properties. The largest effect was evident in the PXRD patterns of the COFs, where a general broadening of the peaks at higher $2 \theta$ was observed moving from $\mathrm{H} \rightarrow \mathrm{CH}_{3} \rightarrow \mathrm{NO}_{2}$ (Figure 1 and Supplementary Figure S3 (bottom)). In addition, the presence of the relatively bulky, flexible and electron-withdrawing $\mathrm{NO}_{2}$ group in $\mathrm{TpPa}-\mathrm{NO}_{2}$, which can potentially block the pore space and result in less-effective $\pi-\pi$ stacking interactions between adjacent layers, afforded lower crystallinity, based on a comparison of the increased intensity of the (001) peak with respect to the (100) peak, which was further demonstrated by the low $S_{\mathrm{BET}}$ even after post-treatment.

To establish the utility of these COFs, we performed a proof-ofprinciple study utilizing post-treated $\mathrm{TpPa}-\mathrm{H}$ as a heterogeneous catalyst to conduct the Suzuki-Miyaura coupling reaction. First, $\mathrm{TpPa}-\mathrm{H}$ was loaded with $\mathrm{Pd}(\mathrm{OAc})_{2}$ to form the $\mathrm{Pd}(\mathrm{OAc})_{2}-\mathrm{TpPa}-\mathrm{H}$ (1) active catalyst (Figure $4 \mathrm{a}) .{ }^{43,44}$ The amount of Pd in $\mathbf{1}$ was determined by inductively coupled plasma atomic emission spectroscopy measurements as $7.06 \mathrm{wt} \%$ (see the corresponding spectrum in Supplementary Figure S14), while the acetate $\mathrm{C}=\mathrm{O}$ resonance at 174 p.p.m. in the ${ }^{13} \mathrm{C}$ cross-polarization magic angle spinning ssNMR spectrum (Figure $4 \mathrm{~b}$ ) confirmed the presence of the $\mathrm{Pd}(\mathrm{OAc})_{2}$ moiety in the framework. In addition, X-ray photoelectron spectroscopy of $\mathbf{1}$ showed characteristic $3 d_{5 / 2}$ and $3 d_{3 / 2}$ peaks at 337.7 and $342.3 \mathrm{eV}$, respectively, in accordance with literature values for a $\mathrm{Pd}^{2+}$ state (Figure 4c). ${ }^{43}$ Note that the characteristic peaks of $\mathrm{Pd}(0)$ metal are observed at $\sim 334.9$ and $340.2 \mathrm{eV} .{ }^{43}$ A comparison of the TGA curves of TpPa-H and $\mathbf{1}$ indicated only a modest loss of structural stability due to $\mathrm{Pd}(\mathrm{OAc})_{2}$ incorporation (Supplementary Figure S15A). Furthermore, 1 displayed a $S_{\mathrm{BET}}$ of $400 \mathrm{~m}^{2} \mathrm{~g}^{-1}$, which decreased compared to $\mathrm{TpPa}-\mathrm{H}\left(632 \mathrm{~m}^{2} \mathrm{~g}^{-1}\right)$, while the pore size distribution curves of $\mathrm{TpPa}-\mathrm{H}$ and 1 indicated $\mathrm{Pd}(\mathrm{OAc})_{2}$ partially occupied the micropores of TpPa-H (Supplementary Figure S15B and C). The $S_{\mathrm{BET}}$ for $\mathbf{1}$ is indicative of fairly accessible pores for potential guests 

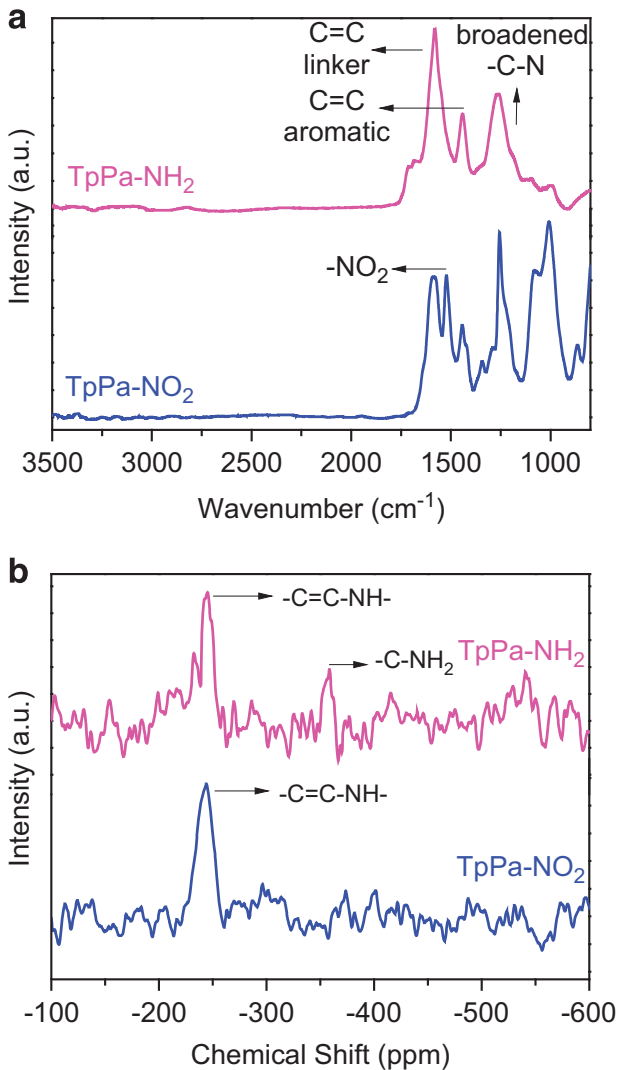

Figure 5 Intra-reduction of $\mathrm{TpPa}-\mathrm{NO}_{2}$ to $\mathrm{TpPa}-\mathrm{NH}_{2}$ via post-synthetic modification in a serial microfluidic process. (a) ATR-IR spectra; (b) ${ }^{15} \mathrm{~N}$ cross-polarization magic angle spinning solid-state nuclear magnetic resonance spectra. Tp, 1,3,5-triformylphloroglucinol.

(Supplementary Figure S15C) when compared to literature reports with other COFs. ${ }^{43,44}$ The catalytic reactions using $\mathbf{1}$ resulted in expected biphenyl product yields in excess of 90\% (Supplementary Table S2 and Supplementary Figures S16-S18). Note that the pristine $\mathrm{TpPa}-\mathrm{H}$ was unable to catalyze these reactions at all. The high surface area of 1 compared to other Pd-loaded COFs and only $\sim 4 \% \mathrm{Pd}$ leaching after three rigorous catalytic cycles $\left(7.06 \mathrm{wt} \% \quad \mathrm{Pd}_{\text {initial }}\right.$ to $6.77 \mathrm{wt} \% \mathrm{Pd}_{\text {cycle3 }}$ in Supplementary Figure S14) could be the result of the strong adherence of $\mathrm{Pd}(\mathrm{OAc})_{2}$ in the post-treated COF layers, which signifies a moderate improvement over the work of Ding et al. ${ }^{44}$ despite our limited scope of reactants under similar conditions.

The next target was to serially modify the obtained COFs into potentially more useful forms, particularly transforming $\mathrm{TpPa}-\mathrm{NO}_{2}$ into $\mathrm{TpPa}-\mathrm{NH}_{2}$. The presence of reactive functional groups, such as $-\mathrm{NH}_{2}$, in framework structures facilitate intended applications, such as selective gas adsorption ${ }^{45}$. However, pre-synthetic $-\mathrm{NH}_{2}$ incorporation in the COFs under study is complicated, as this functionality strongly reacts with the aldehydic OBUs, which even inhibits framework formation. ${ }^{3}$ Therefore, a separate post-synthetic modification process is often required, which entails step-wise purification and separation steps. To avoid these issues, we applied a one-step, serial microfluidic strategy to reduce $\mathrm{TpPa}-\mathrm{NO}_{2}$ to $\mathrm{TpPa}-\mathrm{NH}_{2}$, which is advantageous in terms of integrated and on-demand syntheses. For this purpose, droplets containing the grown $\mathrm{TpPa}-\mathrm{NO}_{2}$ particles were directly fed into another T-micromixer, where they spontaneously merged with a $\mathrm{SnCl}_{2}$ solution in DMA/EtOH (9:1, v/v) after careful optimization of the flow rates (Figure $1 \mathrm{~b}$ ). The merged droplets moved through a coiled capillary in a heating bath maintained at $50^{\circ} \mathrm{C}$ for only $10 \mathrm{~min}$.
This serial microfluidic strategy did not require any protracted separation steps and completed the transformation in 1/10th of the reported time of the bulk process. ${ }^{15}$

This intra-reduction process was monitored by ATR-IR analysis, where characteristic $-\mathrm{NO}_{2}$ resonances (vide supra) disappeared and a broader $\mathrm{C}-\mathrm{N}$ resonance $\left(-\mathrm{C}-\mathrm{NH}_{2}\right)$ appeared at $1261 \mathrm{~cm}^{-1}$ (Figure 5a). Complementarily, the ${ }^{15} \mathrm{~N}$ cross-polarization magic angle spinning ssNMR spectra of $\mathrm{TpPa}-\mathrm{NO}_{2}$ showed a single signal at -244 p.p.m. corresponding to a $2^{\circ}$ amine $(-\mathrm{C}=\mathrm{NH})$, while $\mathrm{TpPa}-$ $\mathrm{NH}_{2}$ exhibited an additional signal at -357 p.p.m. assigned to the $1^{\circ}$ amine $\left(-\mathrm{C}-\mathrm{NH}_{2}\right)$ in the framework (Figure $5 \mathrm{~b}$ ). Furthermore, there were no discernable changes in the PXRD patterns, crystallinities and morphologies of the COFs after the reduction process (Supplementary Figures S19 and S20). Interestingly, the $S_{\mathrm{BET}}$ for $\mathrm{TpPa}-\mathrm{NH}_{2}$ was found to be $177 \mathrm{~m}^{2} \mathrm{~g}^{-1}$, slightly higher than that of $\mathrm{TpPa}-\mathrm{NO}_{2}\left(167 \mathrm{~m}^{2} \mathrm{~g}^{-1}\right)$ (Supplementary Figure S21). These analyses imply that the substituent chemistry was selectively and precisely switched throughout the framework, which was made possible by the fairly accessible pores in the $\mathrm{TpPa}-\mathrm{NO}_{2}$ backbone, with no change in structural integrity in our serially intensified synthesis. Therefore, we conclude that our onestep synthesis and post-modification strategy via this model reaction is effective and time efficient. To the best of our knowledge, this is the first report on a serially intensified synthesis and post-synthetic modification of COFs in a CFM technique.

\section{CONCLUSIONS}

To summarize, our objective was to develop a fast, effective and serial process for the synthesis of COFs and their post-synthetic modification by to avoid long separation and purification steps. For this purpose, we confined OBUs for the synthesis of specially chosen $\beta$-ketoenamine-linked COFs in continuously moving droplets in a transparent capillary for $15 \mathrm{~min}$ at $100{ }^{\circ} \mathrm{C}$ and obtained crystalline COFs, as established by complementary techniques. The choice of solvent, which showed very limited volatility at high temperature $\left(\sim 100^{\circ} \mathrm{C}\right)$ and allowed only partial solubility of the reactant(s), was the critical factor for a stable, continuous stream of microdroplets. However, the choice of solvent may vary for different classes of COFs, and this could restrict the feasibility of our process in limited cases. In addition, the flow rate ratio of the carrier fluid and reactant solution played a considerable role in the creation of microdroplets at the T-micromixer. We speculate that a simple post-treatment could help reduce stacking faults and improve the COF properties.

To assess the applicability of the continuously synthesized COFs, we used $\mathrm{Pd}(\mathrm{OAc})_{2}$-loaded TpPa-H as an active catalyst to perform proofof-principle catalysis of the Suzuki-Miyaura coupling reaction. It is noteworthy that the in-line post-synthetic modification of these COFs, as established by the conversion of $\mathrm{TpPa}-\mathrm{NO}_{2}$ to $\mathrm{TpPa}-\mathrm{NH}_{2}$ via a serial microfluidic approach, is a promising step forward to eliminate the long separation and purification steps to realize mass production of suitably functionalized COFs for desired activities.

\section{CONFLICT OF INTEREST}

The authors declare no conflict of interest.

\section{ACKNOWLEDGEMENTS}

We gratefully acknowledge the support from the National Research Foundation (NRF) of Korea funded by the Korean government (MSIP)

(NRF-2017R1A3B1023598) and the Robot Industry Fusion Core Technology Development Project through the Korea Evaluation Institute of Industrial Technology (KEIT) funded by the Ministry of Trade, Industry and Energy of Korea (MOTIE) (Grant No. 10052980). 


\section{PUBLISHER'S NOTE}

Springer Nature remains neutral with regard to jurisdictional claims in published maps and institutional affiliations.

1 Côté, A. P., Benin, A. I., Ockwig, N. W., O’Keeffe, M., Matzger, A. J. \& Yaghi, O. M Porous, crystalline, covalent organic frameworks. Science 310, 1166-1170 (2005).

2 Waller, P. J., Gándara, F. \& Yaghi, O. M. Chemistry of covalent organic frameworks. Acc. Chem. Res 48, 3053-3063 (2015).

3 Diercks, C. S. \& Yaghi, O. M. The atom, the molecule, and the covalent organic framework. Science 355, eaal1585 (2017).

4 Huang, N., Chen, X., Krishna, R. \& Jiang, D. Two-dimensional covalent organic frameworks for carbon dioxide capture through channel-wall functionalization. Angew. Chem. Int. Ed. 54, 2986-2990 (2015).

5 Lin, S., Diercks, C. S., Zhang, Y.-B., Kornienko, N., Nichols, E. M., Zhao, Y., Paris, A. R., Kim, D., Yang, P., Yaghi, O. M. \& Chang, C. J. Covalent organic frameworks comprising cobalt porphyrins for catalytic $\mathrm{CO}_{2}$ reduction in water. Science 349, 1208-1213 (2015).

$6 \mathrm{Xu}, \mathrm{H} ., \mathrm{Gao}, \mathrm{J}$. \& Jiang, D. Stable, crystalline, porous, covalent organic frameworks as a platform for chiral organocatalysts. Nat. Chem. 7, 905-912 (2015).

7 Vyas, V. S., Haase, F., Stegbauer, L., Savasci, G., Podjaski, F., Ochesenfeld, C. \& Lotsch, B. V. A tunable azine covalent organic framework platform for visible lightinduced hydrogen generation. Nat. Commun. 6, 8508 (2015).

8 Li, Z., Zhi, Y., Feng, X., Ding, X., Zou, Y., Liu, X. \& Mu, Y. An azine-linked covalent organic framework: synthesis, characterization and efficient gas storage. Chem. Eur. J. 21, 12079-12084 (2015).

9 Kharul, U. K., Banerjee, R., Biswal, B. \& Chaudhari, H. D. Chemically stable covalent organic framework (COF)-polybenzimidazole hybrid membranes: enhanced gas separation through pore modulation. Chem. Eur. J. 22, 4695-4699 (2016).

10 Ding, S.-Y., Dong, M., Wang, Y.-W., Chen, Y.-T., Wang, H.-Z., Su, C.-Y. \& Wang, W. Thioether-based fluorescent covalent organic framework for selective detection and facile removal of mercury (II). J. Am. Chem. Soc. 138, 3031-3037 (2016).

11 Lin, G., Ding, H., Yuan, D., Wang, B. \& Wang, C. A pyrene-based, fluorescent threedimensional covalent organic framework. J. Am. Chem. Soc. 138, 3302-3305 (2016).

12 Bunck, D. N. \& Dichtel, W. R. Internal functionalization of three-dimensional covalent organic frameworks. Angew. Chem. Int. Ed. 51, 1885-1889 (2012).

13 Fang, Q., Zhuang, Z., Gu, S., Kaspar, R. B., Zheng, J., Wang, J., Qui, S. \& Yan, Y. Designed synthesis of large-pore crystalline polyimide covalent organic frameworks. Nat. Commun. 5, 4503 (2014).

14 Kandambeth, S., Shinde, D. B., Panda, M. K., Lukose, B., Heine, T. \& Banerjee, R. Enhancement of chemical stability and crystallinity in porphyrin-containing covalent organic frameworks by intramolecular hydrogen bonds. Angew. Chem. Int. Ed. 52 , 13052-13056 (2013)

15 Lohse, M. S., Stassin, T., Naudin, G., Wuttke, S., Ameloot, R., Vos, D. D., Medina, D. D. \& Bein, T. Sequential pore wall modification in a covalent organic framework for application in lactic acid adsorption. Chem. Mater. 28, 626-631 (2016).

16 Huang, N., Krishna, R. \& Jiang, D. Tailor-made pore surface engineering in covalent organic frameworks: systematic functionalization for performance screening. J. Am. Chem. Soc. 137, 7079-7082 (2015).

17 Feng, X., Ding, X. \& Jiang, D. Covalent organic frameworks. Chem. Soc. Rev. 41 6010-6022 (2012).

18 Garzón-Tovar, L., Rodríguez-Hermida, S., Imaz, I. \& Maspoch, D. Spray drying for making covalent chemistry: postsynthetic modification of metal-organic frameworks. J. Am. Chem. Soc. 139, 897-903 (2017).

19 Islamoglu, T., Kim, T., Kahveci, Z., El-Kadri, O. M. \& El-Kaderi, H. M. Systematic postsynthetic modification of nanoporous organic frameworks for enhanced $\mathrm{CO}_{2}$ capture from flue gas and landfill gas. J. Phys. Chem. C 120, 2592-2599 (2016).

20 Ren, S., Bojdys, M. J., Dawson, R., Laybourn, A., Khimyak, Y. Z., Adams, D. J. \& Cooper, A. I. Porous, fluorescent, covalent triazine-based frameworks via roomtemperature and microwave-assisted synthesis. Adv. Mater. 24, 2357-2361 (2012).

21 Campbell, N. L., Clowes, R., Ritchie, L. K. \& Cooper, A. I. Rapid microwave synthesis and purification of porous covalent organic frameworks. Chem. Mater. 21, 204-206 (2009).

22 Chandra, S., Kandambeth, S., Biswal, B. P., Lukose, B., Kunjir, S. M., Chaudhary, M., Babarao, R., Heine, T. \& Banerjee, R. Chemically stable multilayered covalent organic nanosheets from covalent organic frameworks via mechanical delamination. J. Am. Chem. Soc. 135, 17853-17861 (2013).

23 Faustini, M., Kim, J., Jeong, G.-Y., Kim, J. Y., Moon, H. R., Ahn, W.-S. \& Kim, D.-P. Microfluidic approach toward continuous and ultrafast synthesis of metal-organic framework crystals and hetero structures in confined microdroplets. J. Am. Chem. Soc. 135, 14619-14626 (2013).

24 Ameloot, R., Vermoortele, F., Vanhove, W., Roeffaers, M. B. J., Sels, B. F. \& Vos, D. E. D. Interfacial synthesis of hollow metal-organic framework capsules demonstrating selective permeability. Nat. Chem. 3, 382-387 (2011).
25 Hoang, P. H., Park, H. \& Kim, D.-P. Ultrafast and continuous synthesis of unaccommodating inorganic nanomaterials in droplet-and ionic liquid-assisted microfluidic system. J. Am. Chem. Soc. 133, 14765-14770 (2011).

26 Zhang, L., Niu, G., Lu, Ning., Wang, J., Tong, L., Wang, L., Kim, M. J. \& Xia, Y. Continuous and scalable production of well-controlled noble-metal nanocrystals in milliliter-sized droplet reactors. Nano Lett. 14, 6626-6631 (2014).

$27 \mathrm{Su}, \quad$ M. Synthesis of highly monodisperse silica nanoparticles in the microreactor system. Korean J. Chem. Eng. 34, 484-494 (2017).

28 Song, H. \& Ismagilov, R. F. Millisecond kinetics on a microfluidic chip using nanoliters of reagents. J. Am. Chem. Soc. 125, 14613-14619 (2003).

29 Colson, J. W., Woll, A. R., Mukherjee, A., Levendorf, M. P., Spitler, E. L., Shields, V. B., Spencer, M. G., Park, J. \& Dichtel, W. R. Oriented 2D covalent organic framework thin films on single-layer graphene. Science 332, 228-231 (2011).

30 Chong, J. H., Sauer, M., Patrick, B. O. \& MacLachlan, M. J. Highly stable keto-enamine salicylideneanilines. Org. Lett. 5, 3823-3826 (2003).

31 Kandambeth, S., Mallick, A., Lukose, B., Mane, M. V., Heine, T. \& Banerjee, R. Construction of crystalline 2D covalent organic frameworks with remarkable chemical (acid/base) stability via a combined reversible and irreversible route. J. Am. Chem. Soc. 134, 19524-19527 (2012).

32 Biswal, B. P., Chandra, S., Kandambeth, S., Lukose, B., Heine, T. \& Banerjee, R. Mechanochemical synthesis of chemically stable isoreticular covalent organic frameworks. J. Am. Chem. Soc. 135, 5328-5331 (2013)

$33 \mathrm{Niu}$, G., Ruditskiy, A., Vara, M. \& Xia, Y. Toward continuous and scalable production of colloidal nanocrystals by switching from batch to droplet reactors. Chem. Soc. Rev. 44 5806-5820 (2015).

34 Theberge, A. B., Courtois, F., Schaerli, Y., Fischlechner, M., Abell, C., Hollfelder, F. \& Huck, W. T. S. Microdroplets in microfluidics: an evolving platform for discoveries in chemistry and biology. Angew. Chem. Int. Ed. 49, 5846-5868 (2010).

35 Thorsen, T., Maerkl, S. \& Quake, S. R. Microfluidic large-scale integration. Science 298, 580-584 (2002)

36 Elvira, K. S., Solvas, X. C. i., Wootton, R. C. R. \& deMello, A. J. The past present and potential for microfluidic reactor technology in chemical synthesis. Nat. Chem. 5, 905-915 (2013).

37 Peng, Y., Wong, W. K., Hu, Z., Cheng, Y., Yuan, D., Khan, S. A. \& Zhao, D. Room temperature batch and continuous flow synthesis of water-stable covalent organic frameworks (COFs). Chem. Mater. 28, 5095-5101 (2016).

38 Bisbey, R. P., DeBlase, C. R., Smith, B. J. \& Dichtel, W. R. Two-dimensional covalent organic framework thin films grown in flow. J. Am. Chem. Soc. 138 , 11433-11436 (2016).

39 Biswal, B. P., Kandambeth, S., Chandra, S., Shinde, D. B., Bera, S., Karak, S., Garai, B., Kharul, U. K. \& Banerjee, R. Pore surface engineering in porous, chemically stable covalent organic frameworks for water adsorption. J. Mater. Chem. 3, 23664-23669 (2015).

40 Guo, J., Xu, Y., Jin, S., Chen, L., Kaji, T., Hondsho, Y., Addicoat, M. A., Kim, J., Saeki, A., Ihee, H., Seki, S., Irle, S., Hiramoto, M., Gao, J. \& Jiang, D. Conjugated organic framework with three-dimensionally ordered stable structure and delocalized $\pi$ clouds. Nat. Commun. 4, 2736 (2013).

41 Vitaku, E. \& Dichtel, W. R. Synthesis of 2D imine-linked covalent organic frameworks through formal transimination reactions. J. Am. Chem. Soc. 139, 12911-12914 (2017).

42 Smith, B. J., Overholts, A. C., Hwang, N. \& Dichtel, W. R. Insight into the crystallization of amorphous imine-linked polymer networks to 2D covalent organic frameworks. Chem. Commun. 52, 3690-3693 (2016).

43 Gonçalves, R. S., Oliveira, A. B. V. d., Sindra, H. C., Archanjo, B. S., Mendoza, M. E., Carneiro, L. S. A., Buarque, C. D. \& Esteves, P. M. Heterogeneous catalysis by covalent organic frameworks (COF): $\mathrm{Pd}(\mathrm{OAc})_{2} @ \mathrm{COF}-300$ in cross-coupling reactions. ChemCatChem 8, 743-750 (2016).

44 Ding, S.-Y., Gao, J., Zhang, Y., Song, W.-G., Su, C.-Y. \& Wang, W. Construction of covalent organic framework for catalysis: Pd/COF-LZU1 in Suzuki-Miyaura coupling reaction. J. Am. Chem. Soc. 133, 19816-19822 (2011).

45 Tanabe, K. K. \& Cohen, S. M. Postsynthetic modification of metal-organic frameworks -a progress report. Chem. Soc. Rev. 40, 498-519 (2011).

This work is licensed under a Creative Commons Attribution 4.0 International License. The images or
other third party material in this article are included in the article's Creative Commons license, unless indicated otherwise in the credit line; if the material is not included under the Creative Commons license, users will need to obtain permission from the license holder to reproduce the material. To view a copy of this license, visit http:// creativecommons.org/licenses/by/4.0/

(C) The Author(s) 2018

Supplementary Information accompanies the paper on the NPG Asia Materials website (http://www.nature.com/am) 